\title{
Performance Investigation of Two AE Source Location Techniques on a Planar Multilayer Structure
}

\author{
Md. Mehedi Hassan \\ Department of Science and Advanced Technology, Saga University, 1 Honjo-machi, Saga 840-8502, Japan.
}

\author{
Md. Tawhidul Islam Khan
}

Faculty of Science and Engineering, Saga University, 1 Honjo-machi, Saga 840-8502, Japan.

\section{Yuji Hasemura}

Department of Advanced Health Sciences, Saga University, 1 Honjo-machi, Saga 840-8502, Japan.

\author{
Md. Mainul Islam \\ School of Mechanical and Electrical Engineering, University of Southern Queensland, West St, Toowoomba, QLD \\ 4350, Australia.
}

\section{(Received 1 July 2019; accepted 3 January 2020)}

The comparisons of an AE source location performance of two widely used methods, time of arrival (TOA) and difference of arrival time (DOAT) have been analyzed in a complex planar multilayered surface. The complex surface has been prepared by covering a steel plate (SM400A) with a multilayered rubber material (Natural Rubber). The purpose of using this structure has been defined to get the comparable AE source location performance in a complicated signal propagation pathway. Both source location techniques have been conducted in a simulated AE wave propagating plane $(300 \mathrm{~mm} \times 200 \mathrm{~mm}$ in size $)$ under Hsu-Nielsen method. Investigations reveal that the TOA technique gives several erroneous results along with good results. However, the DOAT method has produced all excellent results, even when using a complex surface. It was found since the TOA method is sensitive to constant velocity continuity, the present complex pathway leads to the velocity discontinuity, and thus, several incorrect results have been found. On the other hand, the DOAT method is dependent on the arrival time difference only, and therefore, it shows a higher performance in the source location, even in the presence of a complex structure.

\section{INTRODUCTION}

Source identification and crack characterization have become the continuous research interest in assessing the mechanical performance of engineering materials. ${ }^{1}$ Multilayered materials have been widely used in industrial applications when corrosion protection, safety, lightweight, etc., features are considered. Monitoring these kinds of complex structures are not as simple as a homogenous structure. Sensing and locating the fault initiation is also tricky due to the restriction of the sensor placement and accurate evaluation for these geometrically complex shapes. Nondestructive Testing (NDT) is a field of engineering encompassing the testing and inspection of material and equipment to evaluate conditions, find flaws and identify defects in order to extend the useful life of the infrastructure. $^{2}$ Various NDT techniques have been well established to detect the surface flaws (by Visual Testing, Magnetic Particle Testing etc.) and internal flaws (by Radiography, Ultrasonic Testing etc.); however, acoustic emission testing (AET) is an essential addition to NDT methods of surveying a structure by monitoring the internal geometric defects as well as the surface geometric defects. The difference between AE and other nondestructive techniques is that $\mathrm{AE}$ can detect the failure activity with its dynamic feature at the very beginning of any minute defect, while other NDT methods detect the flaw at its matured stage. $^{3}$

In acoustic emission monitoring, the source location is possibly a promising aspect. Locating the source of significant acoustic emissions (AEs) is often the primary goal of an AE inspection. Since the majority of the sources of AEs are damagerelated, the sensing and monitoring of these emissions are ordinarily used to anticipate material failure. ${ }^{4,5}$ Detection and analysis of AE signals can supply valuable information regarding the origin and importance of a discontinuity in a material. As acoustic emissions can result from the initiation and growth of cracks; therefore, in AE testing, the source location is an essential paramount tool. The researchers have introduced several AE source location techniques such as Linear Location Technique, 2-dimensional (2D) Location Technique, 3-dimensional (3D) Tomography Technique, Delta $T$ Technique etc. ${ }^{5-10}$

In industrial monitoring, source location algorithms are useful in identifying the damaged location as well as damage characteristics. Pencil-lead breaks (PLB) have been used as a reproducible broadband source (Hsu-Nielsen source) for test signals in AE applications. ${ }^{11}$ The original works of Hsu and Nielsen were conducted with Cliff Bailey at Lockheed Georgia on the surface of an aircraft panel. They used the simulated 
acoustic emission source generated by the breaking of a pencil lead. Since then the PLB technique is often referred to as Hsu-Nielsen source. ${ }^{12-14}$

TOA method that uses the velocity of the wave propagation directly is one of the conventional ways of determining the source of the damaged region, especially for simple structures. However, theoretical wave path analysis becomes more complicated when structures are geometrically complex. Exact arrival time is crucial in order to reduce source location errors. First threshold crossing (FTC), maximum amplitude (MA) and so on, are commonly used methods of picking the arrival time. Since, AE is a complicated wave combined with multiple wave modes ( $\mathrm{p}$-wave, s-wave and so on); consideration of MA in source location of a complicated surface may increase errors due to the different velocity modes of p-wave, s-wave etc. On the other hand, in the FTC method, the onset time of $\mathrm{p}$-wave, which is faster than s-wave can be correctly identified, and thus, uncertainty in source location can be reduced.

However, if there is a high noise in the signal, there is a possibility of having errors in picking the onset time by using the FTC method. In that case, for the determination of the correct onset time, modelling the signal as an autoregressive process (AR) is an alternative approach. ${ }^{15}$ According to that, assumptions signals are divided into two different stationary processes, one is before the onset and the other one is after the onset. ${ }^{16}$ Therefore, Akaike information criterion (AIC) based on autoregressive assumptions are used in seismology to identify $\mathrm{P}$ and $\mathrm{S}$ phases of the waves. ${ }^{17}$ The autoregressive AIC (AR-AIC) algorithm for onset detection is robust, computationally fast and could be implemented automatically.

The present research has been conducted on a complex multilayered structure (a steel plate covered with rubber layers) as an experimental understanding regarding the efficiency of AE source location technique for the validation of the methods for industrial applications. However, in the case of multilayered, complicated structures, like hydrogen fuel storage tank in fuel cell vehicles, etc., AE monitoring for internal damage is complicated and source location becomes unavoidable from the outer surface. Therefore, the objective of the present research by using this complex structure is to gain comparable AE source location performance and suitable source location methods for applicability in a complicated signal propagation pathway. Attachment of extra layers on a structural material makes the signal propagation complicated in the present research. The AE wave propagation encounters the resistance in terms of impedance when it passes through the different material boundaries. The typical source characteristic is, therefore, quite complex and can be further confused by reflection, mode conversion and attenuation of waves as the source disturbance propagates to the sensor. ${ }^{18}$

Several recent methods like Bayesian approach and Dong's velocity-free localization approach take the velocity of the wave as an additional unknown parameter and solve it with a source coordinate. ${ }^{19,20}$ The collaborative localization method that uses analytical and iterative solutions for microseismic/acoustic emission sources, can filter the abnormal arrivals and improve the accuracy of the source location as well. ${ }^{21}$ Nevertheless, the difference of arrival time (DOAT) method, similar to the Delta $T$ method as described by Baxter et al., records arrival time differences from several locations, for op- timizing source location of the complex, multilayered structure. ${ }^{10}$ This method does not depend on wave velocity measurement. It uses only the arrival time difference in source location calculation. Therefore, this method has an advantage in calculating source location over the TOA method. In the current paper, the DOAT method has been applied on a planar steel structure covered with multilayers of rubber material. Comparative analysis of an efficient source location between TOA and DOAT methods have been conducted as well. The accurate applicability of AE source location investigation demonstrates that the DOAT method is a credible method compared to the TOA method in source location of complex structures.

Section two discusses relevant theories and procedures for AE experiments in wave velocity determination and source location. Section three presents the experimental results and discussion. Finally, section four concludes the present research.

\section{EXPERIMENTAL METHODOLOGY}

\subsection{Determination of Signal Arrival Times}

The determination of TOA has a significant influence on the localization accuracy regardless of any method. FTC and the time of the maximum signal amplitude (MA) are commonly used methods in AE source location technique. ${ }^{22}$ The MA method is somewhat dependent on the signal amplitude at a particular sensor position. Therefore, it is influenced by the sensor distance as well as wave velocity modes. Furthermore, sometimes, it shows the multiple MA, and hence, it becomes challenging to choose the correct one. Thus, the FTC method has been chosen for the acquisition of accurate onset time (Fig. 1). All data acquisition in the present experiment has been conducted in a noise-free environment with controlled temperature. To determine the time of arrival through the FTC method, an appropriate constant threshold value of 0.08 volt has been applied based on the condition of acquisition data with a mean zero condition and thus, picks the time of the signal that crosses the threshold value first.

However, if there is a high signal to noise ratio, FTC method might be unreliable in picking the correct onset time. In that case, the onset of a microseismic signal might be determined by modelling the signal as an autoregressive process. It is based on the assumptions that the time series can be divided into two locally stationary processes which initiate with lowamplitude random noise followed by a high-amplitude impulse containing the first arrival of $\mathrm{AE}$ event. ${ }^{23}$ This approach is known as autoregressive Akaike information criterion (ARAIC) and has been used in seismology to detect $P$ and $S$ phases of the wave. Akaike's information criterion was developed in 1973 and later modified by Maeda which is recently used by the researchers in $\mathrm{AE}$ onset detection. ${ }^{24,25}$ For time series $\mathrm{x}$ of length $\mathrm{N}$, the AIC is defined as

$$
\begin{aligned}
A I C(k)= & k \log (\operatorname{var}(x[1, k]))+ \\
& (N-k-1) \log (\operatorname{var}(x[k+1, N])) ;
\end{aligned}
$$

where $k$ is the range through all signal points and $\operatorname{var}(x[1, k])$ indicates the variance of the corresponding interval from 1 to $k$ of signal $x$. The AIC global minimum determines the arrival time. This method of onset picking is also used in the current paper and compared with the FTC method in TOA source location calculations. 


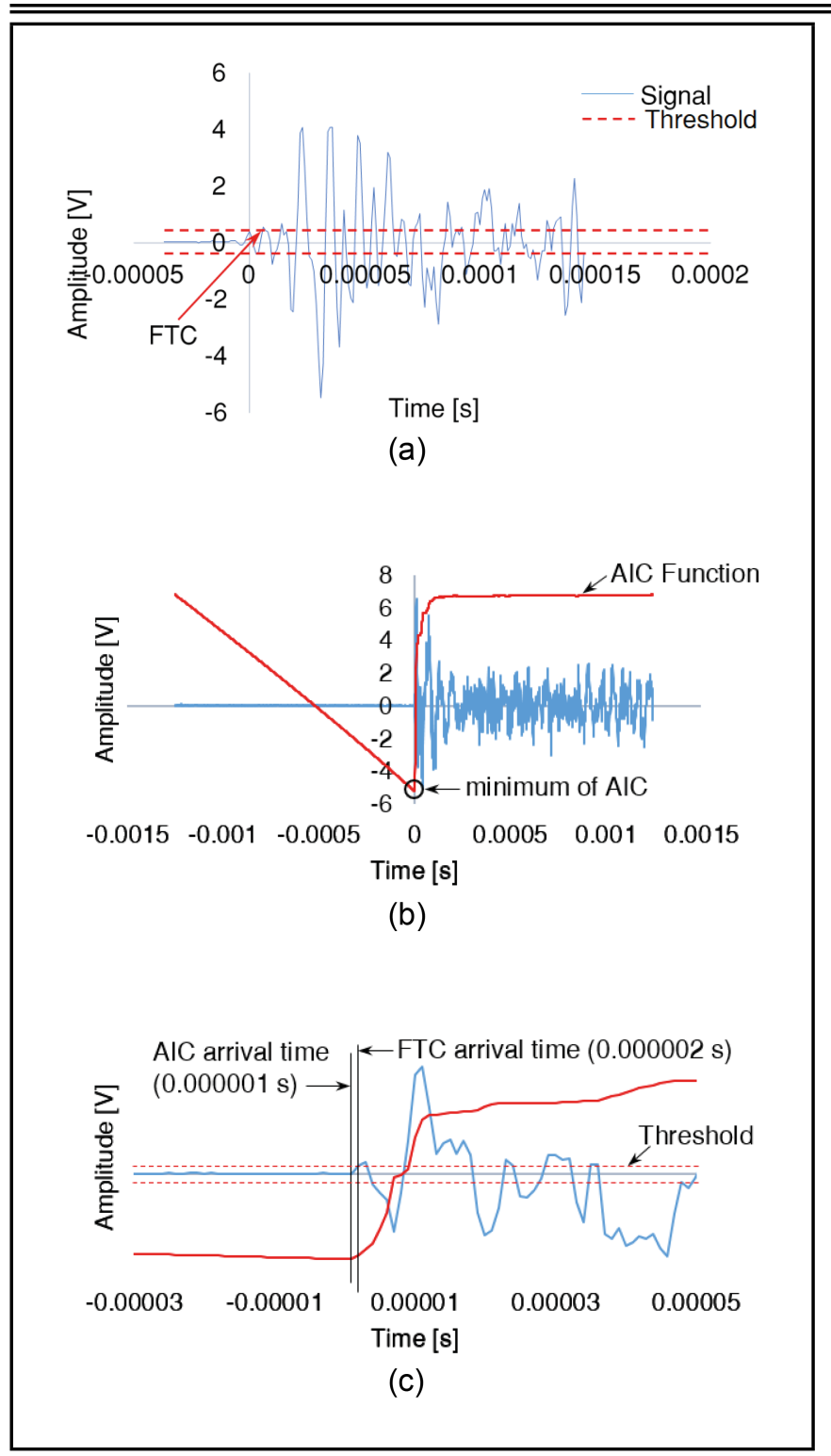

Figure 1. Methods for first arrival time acquisition; (a) FTC method, (b) AIC method and (c) comparison of first arrival time acquisition in FTC and AIC methods for a sample calculation (minimized time window of a sample signal).

\subsection{Wave Velocity Determination}

To compute a source location by TOA method in the AE technique, the velocity of the wave propagation is a crucial factor. $^{26,27}$ The determination of the source location could be affected if incorrect wave velocity is used. ${ }^{28,29}$ Conventionally, two sensors are placed on the specimen in a straight line, and a PLB is performed in line with these sensors. Since the travel distance and the time it takes for the waves to cross this distance are known, the travel speed can be calculated.

This method was used in this paper in order to determine the wave speed. In the present experiment, three AE sensors were attached upon the specimen according to the 2-dimensional (2D) source location algorithm. One sensor was attached below the specimen as a reference sensor which moves at each PLB points for getting corresponding TOA. ${ }^{30}$ PLB tests were performed below and the opposite side of the steel plate at the 17 node points where future TOA source location will be carried on. A total of three PLB tests were conducted at each point to get the average $\mathrm{AE}$ excitation of that point. The breaking place and conditions were kept the same in all of those three

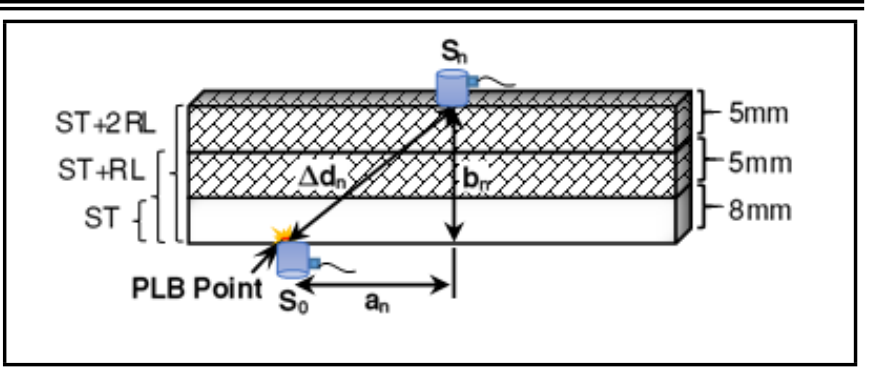

Figure 2. Schematics of the velocity measurement in PLB tests; steel plate (ST) covered with a single rubber layer $(\mathrm{ST}+\mathrm{RL})$ and a double rubber layer (ST+2RL).

tests. Velocities were calculated using the following equations.

$$
\begin{gathered}
v=\frac{\Delta d_{n}}{\Delta t_{n}}=\frac{S_{n}-S_{0}}{t_{n}-t_{0}}, \quad n=1,2,3 ; \\
\Delta d_{n}=\sqrt{{a_{n}{ }^{2}+b_{n}}^{2}} ;
\end{gathered}
$$

where $S_{0}=0$ is the pencil lead breaking point (reference point), $v=$ wave velocity, $\Delta d_{n}=$ distance difference between reference sensor $\left(S_{0}\right)$ and other sensors $\left(S_{n}\right), \Delta t_{n}=$ time difference between the reference sensor $\left(t_{0}\right)$ and other sensors $\left(t_{n}\right), a_{n}=$ distance due to projection of $\Delta d_{n}$ and $b_{n}=$ thickness of the material.

Similarly, two experiments were done keeping the methodology the same covering the steel plate with single and double rubber layers. In all experiments, silica gel was used for avoiding any air gap between plate to rubber layers. The positions of sensors, specifications of the steel plate and rubber layers and the place of pencil lead fracture are shown in Fig. 2.

As explained above, present research contained an extra single and double layer rubber upon the steel specimen, which made the signal propagation complex. AE sensors recorded $\mathrm{AE}$ signals from the rubber surface, which were generated on the steel surface. When the AE signal passes through the different material boundaries, it encounters the impedance effect. ${ }^{2} \mathrm{AE}$ waves are reflected at boundaries due to the difference in acoustic impedances of different materials (steel and rubber). For the multilayer complex structure, signals are obviously largely reflected, however, for simplicity in calculations it is considered that the propagation occurred in a straight line (Fig. 2), and these relations are shown in Eqs. (2) and (3).

\subsection{TOA Source Location}

The widely used method of AE source location is the TOA method. Tobias explained this 2-dimensional (2D) source location method in details. ${ }^{5,8,31}$ In the present analysis, the TOA method was applied as well. In this method, three sensors were used for the calculation of the source location. The schematics of sensor arrangements in a triangular setup with sensor spacing algorithm is shown in Fig. 3.

Three sensors $S_{o}(0,0), S_{i}\left(x_{i}, y_{i}\right), S_{j}\left(x_{j}, y_{j}\right)$ were used to compute 2D AE source location as in Fig 3. $S\left(x_{s}, y_{s}\right)$ was the AE source, which was calculated from the reference sensor $S_{o}$ at a distance of $r_{s}$ and at an angle of $\theta$.

The time difference of arrival (TDOA) $\Delta t_{i j}$ of a signal generated by a point source $S$ and detected at sensors $i$ and $j$ can be written as:

$$
\Delta t_{i j}=t_{i}-t_{j}
$$




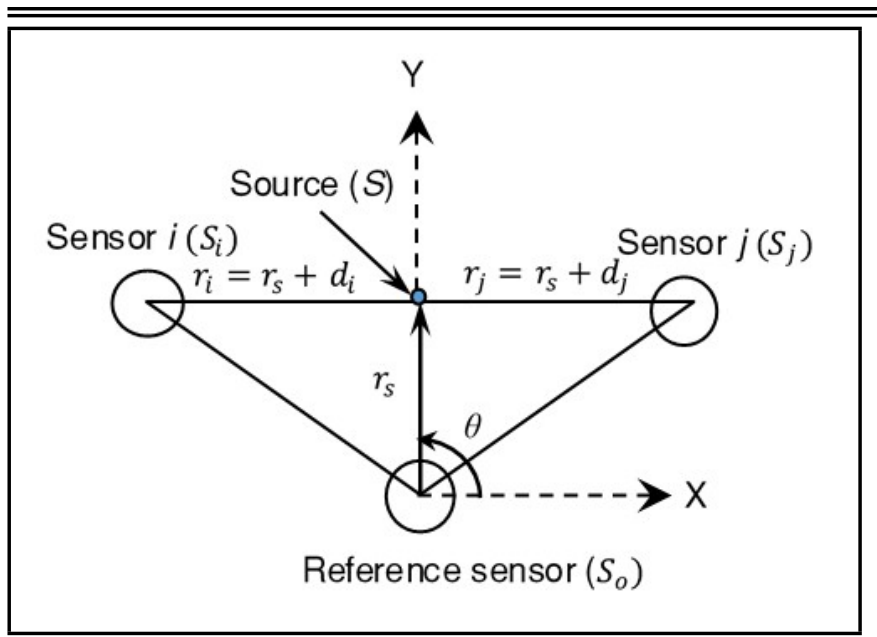

Figure 3. Schematics of sensor arrangements in a triangular setup for the investigation of TOA source location.

where, $t$ is the ideal propagation time from source to the sensor and the subscripts $i, j$ denote different sensors. Thus, for changing locations concerning changes of $S_{o}$, TDOA from the source were defined by: $\Delta t_{i o}=t_{i}-t_{o}$ or $\Delta t_{j o}=t_{j}-t_{o}$.

Therefore, the difference in distance between source and sensors are:

$$
\begin{gathered}
d_{i}=r_{i}-r_{s}=v \cdot \Delta t_{i o} \\
d_{j}=r_{j}-r_{s}=v \cdot \Delta t_{j o}
\end{gathered}
$$

where $v$ is the velocity of wave propagation in the material and $\Delta t_{i o}, \Delta t_{j o}$ are TDOA for sensors $\left(S_{i}-S_{o}\right)$ and $\left(S_{j}-S_{o}\right)$ respectively. The algorithm for determining two-dimensional source location are explained as follows:

$$
\begin{gathered}
r_{s}=\frac{1}{2}\left[\frac{x_{i}^{2}+y_{i}^{2}-d_{i}^{2}}{x_{i} \cos \theta+y_{i} \sin \theta+d_{i}}\right]= \\
\frac{1}{2}\left[\frac{x_{j}^{2}+y_{j}^{2}-d_{j}^{2}}{x_{j} \cos \theta+y_{j} \sin \theta+d_{j}}\right] ; \\
\theta=\tan ^{-1}\left(\frac{U_{i} y_{j}-U_{j} y_{i}}{U_{i} x_{j}-U_{j} x_{i}}\right)+ \\
\cos ^{-1}\left|\frac{U_{j} d_{i}-U_{i} d_{j}}{\left[\left(U_{i} x_{j}-U_{j} x_{i}\right)^{2}+\left(U_{i} y_{j}-U_{j} y_{i}\right)^{2}\right]^{1 / 2}}\right| ;
\end{gathered}
$$

where, $U_{i}=x_{i}^{2}+y_{i}^{2}-d_{i}^{2}$ and $U_{j}=x_{j}^{2}+y_{j}^{2}-d_{j}^{2}$,

$$
x_{s}=r_{s} \cos \theta \text { and } y_{s}=r_{s} \sin \theta \text {. }
$$

By employing Eqs. (7), (8) and (9), 2D source location was calculated. From the above analysis, it was found that the TOA method is greatly influenced by the wave propagation velocity.

Artificial AE events were generated in the experiment based on standards of Hsu-Neilsen source (H-N source) technique by PLB tests. ${ }^{8}$ Three different types of experiments were conducted by changing the test section as follows:

1. Case 1: On the surface of the steel specimen;

2. Case 2: On the surface of the steel specimen covered with single rubber layer; and,
3. Case 3: On the surface of the steel specimen covered with double rubber layer.

PLB tests were conducted in a noise-free environment from below and the opposite side of the steel surface (test region) at 17 different places (randomly selected) for all of the three cases mentioned above. PLB kept at the same places in all cases. According to the above 2D source location algorithm, three sensors were placed surrounding the PLB points (source points) and source distance $\left(r_{s}\right)$ and inclination $(\theta)$ of $r_{s}$ (Fig. 3) were calculated by applying conventional three circle equations considering the corresponding co-ordinates of three sensor positions and sensor distances, respectively. The solutions of these equations were determined considering the source point of $\mathrm{AE}$ signals (PLB point) as the co-incident point of all those three circle equations. After solving those criteria, 2D AE source was calculated by the values of $r_{s}$ and $\theta$.

\subsection{DOAT Source Location}

The operation of the DOAT method is based on the arrival time differences (ATDs) of signals received by the pair of sensors. The idea is to establish a DOAT database map measuring the ATDs of AE signals generated by pencil-lead breaks throughout the test area. Several steps associated with this location method are described below.

Step 1, Test area for sensor placement: The initial step of this method is to define the test area (area of interest) and place the sensors around that area. In the present experiment, four sensors were placed at each corner of the test area of $300 \mathrm{~mm}$ in length, $200 \mathrm{~mm}$ in width upon a large steel plate (SM400A) having a dimension of $611 \mathrm{~mm}$ in length, $507 \mathrm{~mm}$ in width and the thickness of the plate was $0.008 \mathrm{~m}(8 \mathrm{~mm})$ as shown in Fig. 4(a). Figure 4(b) represents detail information of the interested area where black dots are PLB nodes for both TOA and DOAT methods and red crossing points are source location verification nodes specifying the coordinates.

Step 2, Create meshes for node points: Once the experimental area is identified, the second step is to divide the area with equal meshes and define node points. For getting better accuracy, a mesh density of $25 \mathrm{~mm}$ was arbitrarily used. It generated 99 nodes numbering $n_{1}, n_{2}, n_{3}, \ldots \ldots \ldots n_{99}$ in this experiment.

Step 3, PLB TOA measurement: The following step is to carry on PLB at each node and extract TOA information from all nodes according to the Eq. (10).

$$
f\left(P L B_{n}\right)=[T O A s]_{n}=\left[\begin{array}{l}
T O A @ \text { sensor } 1 \\
T O A @ \text { sensor } 2 \\
T O A @ \text { sensor } 3 \\
T O A @ \text { sensor } 4
\end{array}\right]_{n}
$$

where, $f(P L B)$ indicates the function of PLB, TOAs indicate the time of arrivals for four sensors and $\mathrm{n}$ stands for the number of the node points.

Step 4, Create DOAT database map: After extracting the TOA information from all the nodes (by Eq. (10)), the DOAT for each sensor pair was estimated. An array of four sensors generated six sensor pairs 1-2, 1-3, 1-4, 2-3, 2-4 and 3-4. The DOAT for sensor pair 1-2 is therefore defined as DOAT = $T O A_{1}-T O A_{2}$. Similarly, the DOAT for other sensor pairs were estimated, and the databases were constructed employing 
the Eq. (11).

$$
f\left(P L B_{n}\right)=[D O A T s]_{n}=\left[\begin{array}{c}
T O A_{1}-T O A_{2} \\
T O A_{1}-T O A_{3} \\
T O A_{1}-T O A_{4} \\
T O A_{2}-T O A_{3} \\
T O A_{2}-T O A_{4} \\
T O A_{3}-T O A_{4}
\end{array}\right]_{n}
$$

Three different tests for three cases were carried out in the present experiment by altering the test section. Three cases are outlined below:

1. Case 1: A mesh density of $25 \mathrm{~mm}$ was used, creating 99 nodes on the surface of the steel specimen;

2. Case 2: A mesh density of $25 \mathrm{~mm}$ was used, creating 99 nodes on the surface of the steel specimen covered with single rubber layer; and,

3. Case 3: A mesh density of $25 \mathrm{~mm}$ was used, creating 99 nodes on the surface of the steel specimen covered with double rubber layer .

In order to provide average results, a total of three PLBs were conducted at each node in each test. According to the objectives of the research, all the PLBs were conducted from the opposite side of the steel surface (test section), so that generated signals travel to sensors through complex pathways.

Step 5, Create contours for source location: From the output of step 4 from the DOAT database, DOAT maps were constructed based on all received $\mathrm{AE}$ events. In each case, six maps were created for the six pairs of sensors. Six sensor pairs (1-2, 1-3, 1-4, 2-3, 2-4, 3-4) generated six DOAT contours as $T O A_{1}-T O A_{2}, T O A_{1}-T O A_{3}, T O A_{1}-T O A_{4}, T O A_{2}-$ $T O A_{3}, T O A_{2}-T O A_{4}, T O A_{3}-T O A_{4}$. Those six DOAT contours generated six DOAT contour maps. For a specific AE event, each map represented a contour line of equal DOAT, and thus six maps constituted six contour lines. A convergence point was identified by overlaying all the six DOAT contour maps, and that convergence point was the desired source location.

\subsection{Experimental Procedure}

An artificial AE was simulated using pencil-lead fractures (Hsu-Neilsen source or H-N source) on a steel plate (SM400A). The dimension of the plate was $611 \times 507 \times 8 \mathrm{~mm}$. The specimen preparation and experimental setup with its operation domain are explained in Fig. 5. The schematic diagram and experimental device diagram are represented in Figs. 5(a) and 5(b) respectively. Four circular general-purpose piezoelectric sensors (Physical Acoustics Corporation, model R15 $\alpha$ with a frequency range of $50-200 \mathrm{kHz}$ as well as a size of $18 \mathrm{~mm}$ in diameter and $17 \mathrm{~mm}$ in thickness) were placed on the steel plate using acoustic coupling gel, one at each corner of the experimental area. Before connecting to the main AE amplifier (four-channel, Japan Physical Acoustics), all the sensors were connected to the respective pre-amplifiers (2/4/6 of Physical Acoustics Corporation) with $40 \mathrm{~dB}$ gain to boost the weak signal into the line level for the amplifier. For the acquisition of $\mathrm{AE}$ events, a four-channel digital oscilloscope (Tektronix TDS 2024C) was connected to the amplifier. By

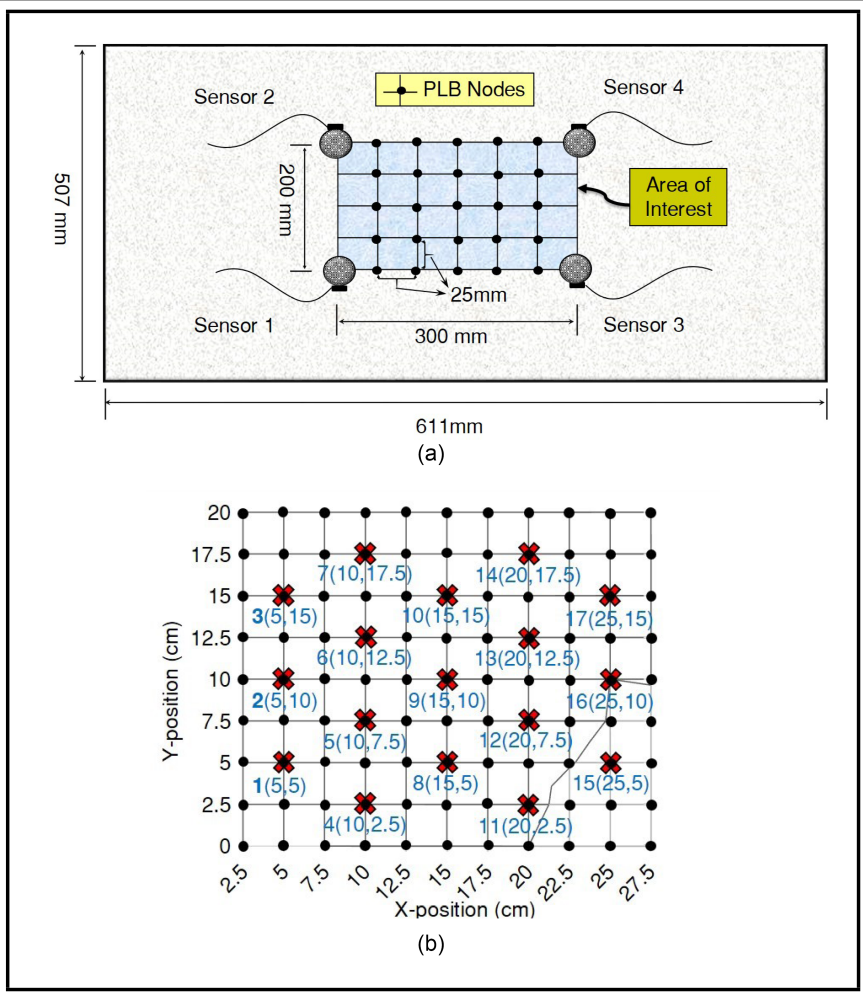

Figure 4. (a) The dimension of the test section with four AE sensors, (b) Source location verification nodes $(1 \sim 17)$ specifying the coordinates (within brackets) on a contour graph.

using the oscilloscope, AE signal features were extracted and stored in the computer for the analysis of AE source location. The sampling frequency was considered as $1 \mathrm{MHz}$. The triggered channel was kept constant.

\section{EXPERIMENTAL RESULTS AND DISCUSSION}

\subsection{Results of TOA Source Location}

TOA in AE events was calculated from the travelling time of the recognized AE signal by both FTC and AIC methods. The velocity of the $\mathrm{AE}$ signals on test materials at each location point was calculated from the relation of dividing sensor to sensor distance by the travelling time, TOA, as previously explained. Averaging was done for each source location from three consecutive PLB tests for getting the accurate travelling velocity of $\mathrm{AE}$ waves.

TOA source locations were calculated based on the referred algorithm, as mentioned earlier. In those experiments, the average time of arrival of the waveforms was recorded from three consecutive tests, and the measured velocity was used for calculating TOA source location. Randomly, the 17 node points were considered in order to calculate source coordinates for all three cases. Out of 17 points, the majority showed good source locations ( $<20 \%$ error); however, several points showed deviated locations $(>20 \%$ error). This deviation might have occurred due to the velocity effect of the wave propagation as the time of arrival relies on the velocity directly. Results of velocity and source location in percent (\%) error for FTC and AIC methods are shown in Table 1 and Table 2, respectively.

It was found that source location was largely influenced by the velocity of the wave propagation. Although it was obvious and therefore results of source location were obtained in terms 
M. M. Hassan, et al.: PERFORMANCE INVESTIGATION OF TWO AE SOURCE LOCATION TECHNIQUES ON A PLANAR MULTILAYER...

Table 1. Results of velocity and source location in percent error for the FTC method.

\begin{tabular}{|c|c|c|c|c|c|c||}
\hline $\begin{array}{c}\text { Location } \\
\text { No. }\end{array}$ & \multicolumn{2}{|c|}{ Steel Plate } & \multicolumn{2}{c|}{$\begin{array}{c}\text { Steel Plate Covered } \\
\text { with a Single Rubber Layer }\end{array}$} & \multicolumn{2}{c|}{$\begin{array}{c}\text { Steel Plate Covered } \\
\text { with a Double Rubber Layer }\end{array}$} \\
\hline 1 & Velocity (m/s) & $\%$ Error & Velocity (m/s) & \% Error & Velocity (m/s) & $\%$ Error \\
\hline 2 & 4339 & 8.04 & 4013.9 & 8.53 & 2891.09 & 5.29 \\
\hline 3 & 5346.9 & $27.42^{*}$ & 3510.6 & $52.72^{*}$ & 3407.58 & $50.84^{*}$ \\
\hline 4 & 5273.4 & 0.57 & 3533.6 & 4.33 & 2891.09 & 6.41 \\
\hline 5 & 4470.2 & 5.67 & 3545.7 & 7.67 & 2877.11 & 19.23 \\
\hline 6 & 4677.1 & 9.13 & 3808.3 & 2.1 & 2875.17 & 11.85 \\
\hline 7 & 4329.7 & $30.96^{*}$ & 3611.4 & $20.36^{*}$ & 2875.17 & 11.73 \\
\hline 8 & 4492.4 & $44.76^{*}$ & 3084.1 & $34.45^{*}$ & 3198.37 & $24.03^{*}$ \\
\hline 9 & 4548.6 & 4.2 & 3963.3 & 5.1 & 2996.66 & 4.55 \\
\hline 10 & 5328.7 & 0.45 & 4101.6 & 1.5 & 2798.35 & 4.19 \\
\hline 11 & 4752.1 & 0.46 & 3881.8 & 16.21 & 2897.92 & 1.98 \\
\hline 12 & 4649.7 & 1.51 & 3927.3 & 0.39 & 2934.35 & 9.24 \\
\hline 13 & 4577.6 & 0.27 & 4116.1 & 3.48 & 2955.51 & 6.9 \\
\hline 14 & 4617.2 & $29.67 *$ & 4237.8 & 11.92 & 2929.76 & $24.39^{*}$ \\
\hline 15 & 4724.5 & 0.17 & 3928.2 & 10.26 & 2938.33 & 4.32 \\
\hline 16 & 5299.4 & 8.69 & 3853.7 & 10.95 & 2868.1 & 17.56 \\
\hline 17 & 5455.3 & 18.12 & 3467.7 & $32.4 *$ & 2883.51 & 14.79 \\
\hline
\end{tabular}

* indicates that percent error is higher than $20 \%$

Table 2. Results of velocity and source location in percent error for the AIC method.

\begin{tabular}{|c|c|c|c|c|c|c||}
\hline $\begin{array}{c}\text { Location } \\
\text { No. }\end{array}$ & \multicolumn{2}{|c|}{ Steel Plate } & \multicolumn{2}{c|}{$\begin{array}{c}\text { Steel Plate Covered } \\
\text { with a Single Rubber Layer }\end{array}$} & \multicolumn{2}{c|}{$\begin{array}{c}\text { Steel Plate Covered } \\
\text { with Double Rubber Layer }\end{array}$} \\
\hline & Velocity & \% Error & Velocity & \%rror & Velocity & Error \\
\hline 1 & 5344.4 & 9.63 & 4371.3 & 0.57 & 3795.9 & 8.82 \\
\hline 2 & 5076.3 & $35.59^{*}$ & 4495.2 & $31.52^{*}$ & 3900.9 & 12.78 \\
\hline 3 & 5118.7 & 18.11 & 4415.7 & 0.41 & 3801.9 & $31.53^{*}$ \\
\hline 4 & 5385.4 & 0.19 & 4734.3 & 4.17 & 4055.2 & 1.53 \\
\hline 5 & 5233.2 & 3.29 & 4668.8 & 7.19 & 3985.5 & 7.47 \\
\hline 6 & 5420.9 & $25.6 *$ & 4296.6 & $22.46^{*}$ & 4113.5 & $20.55^{*}$ \\
\hline 7 & 5222.33 & $37.66^{*}$ & 4560.4 & $34.38^{*}$ & 4103.3 & $35.78^{*}$ \\
\hline 8 & 5391.2 & 1.31 & 4298.1 & 0.47 & 4104.2 & 5.23 \\
\hline 9 & 5229.5 & 2.5 & 4357 & 1.96 & 4166.2 & 1.3 \\
\hline 10 & 5105.1 & $25.35^{*}$ & 4562.6 & $23.91^{*}$ & 4015.9 & $25.12^{*}$ \\
\hline 11 & 5377.4 & 7.51 & 4427.2 & 1.6 & 4109.1 & 1.06 \\
\hline 12 & 5186 & 4.37 & 4509.3 & 0.41 & 3741.4 & 18.01 \\
\hline 13 & 5087.7 & 3.76 & 4518.6 & 4.45 & 3887.6 & 19.66 \\
\hline 14 & 5200.8 & $25.87^{*}$ & 4667.6 & $22.68^{*}$ & 3948.9 & 19.61 \\
\hline 15 & 5102.5 & 4.06 & 4534.5 & 2.65 & 4003.7 & 2.77 \\
\hline 16 & 5214.9 & 4.42 & 4463.5 & 1.38 & 4151.4 & 6.77 \\
\hline 17 & 5198.9 & 4.89 & 4644.3 & 1.72 & 4113.4 & 6.29 \\
\hline$*$ indicates that percent error is higher than $20 \%$ & & & \\
\hline \multicolumn{7}{|c|}{} \\
\hline
\end{tabular}

of errors $(<20 \%)$ for all conditions of experiments (steel plate, steel plate covered with single rubber layer and steel plate covered with double rubber layer) and are summarized in the following tables. In all three conditions, for both FTC and AIC methods, errors were increased more than $20 \%$ at node points $14,35,52,71$ (equivalent location number $2,7,10,14$ respectively) and their nearby points, particularly, in the steel plate. It was also observed that velocities of those points were also varied, as shown in Figs. 6(a) and 6(b). Those node points have been shown with the circle, the diamond and the square markings for better understanding (Figs. 6(a) and 6(b)). It was considered that various uncertainties could be involved due to some increase in structural inhomogeneity, and therefore, signal scattering in the steel plate might increase the variations in wave velocity measurement as well as source location. Therefore, higher errors might be received in source location at those node points. Those effects were observed in the steel plate covered with a single rubber layer and the steel plate covered with double rubber layer as well. Since PLB tests were con- ducted on the steel plate, those effects were observed higher in the steel plate compared to the steel plate with a single rubber layer and the steel plate with a double rubber layer. Although in both methods (FTC and AIC), source location errors were fluctuated from point to point, overall errors were decreased in the case of AIC methods. Velocity distributions were also less fluctuating in the case of the AIC method.

\subsection{Results of DOAT Source Location}

In response to all sensors to the artificial H-N source (PLB), the time of arrival at each sensor was recorded for each PLB source event. The DOAT for each pair was calculated in order to locate an actual AE event. The average DOAT for each sensor pair at each node was stored in a DOAT database mapping system. The maps can be displayed as contour plots of equal DOAT (Fig. 7). Figures 7(a)-(d) represent the DOAT contour maps for the steel plate, steel plate covered with a single rubber layer and the steel plate covered with double rubber layer respectively. The contour map illustrates that throughout the 


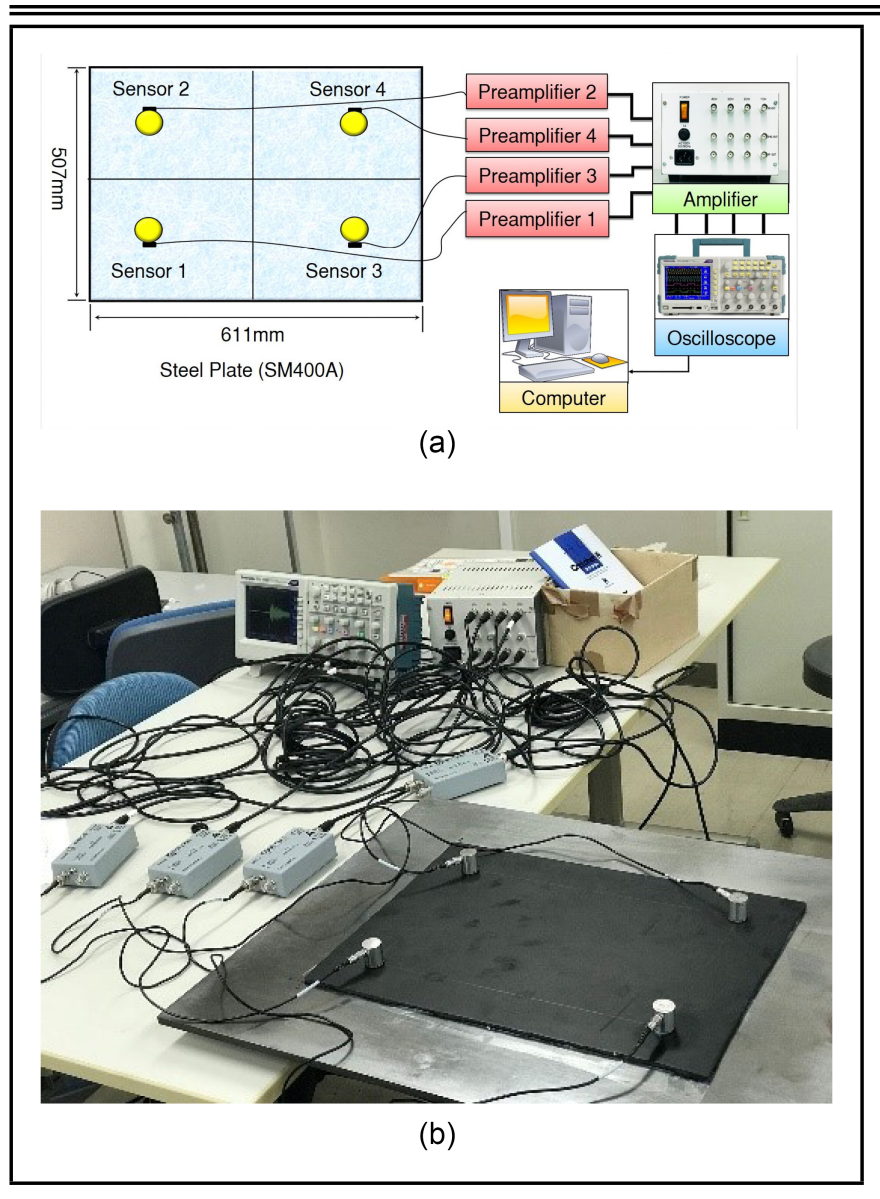

Figure 5. Schematics of the experimental setup with and its operation domain; (a) schematic diagram, (b) experimental device diagram.

contour line, the time difference is equal for the specific pair of sensors. A total of 18 DOAT contour maps (six in each case) were drawn and three samples from all three individual cases [Figs. 7(a)-(c)] are presented here. Figure 7(d) shows the DOAT for the sensor pair 2-4 from a specific AE event, and the contour line (DOAT $39 \mu$ s line) displays the possible source location passing the node point $84\left(n_{84}\right)$ which is equivalent to the source verification coordinate of $15(0.25 \mathrm{~m}, 0.05 \mathrm{~m})$ in Figs. 7 and 8.

Similarly, other five DOAT contour lines were created for the same event, and those lines also showed the possible source location which passed the node point $84\left(n_{84}\right)$. Overlapping all six possible source location contour lines, one above another, from each of the sensor pairs, a convergence point was identified. That was the required source location (Fig. 8). This procedure was repeated for the complete data set of 17 different randomly selected node points.

Figures 8(a)-(c) illustrate the contour graphs from all the six DOAT maps for all combinations of the sensor pairs. Six colors represent six contour lines and all those contour lines intersected at one location, which indicated the location of the source. The figures depict only one location $\left(n_{84}\right)$ from all three individual cases. Likewise, the other 16 places have been calculated in each case and represented in section 3.3. Theoretically, all lines are supposed to coincide at one location, however, in a few cases, all lines did not coincide at the same point especially for the steel plate covered with a double rubber layer $\left(n_{84}\right.$, Fig. 8(c)). It was assumed that in the case of the steel plate covered with a double rubber layer, signal scattering (impedance effect) might occur. For example, in Fig. 8(c),

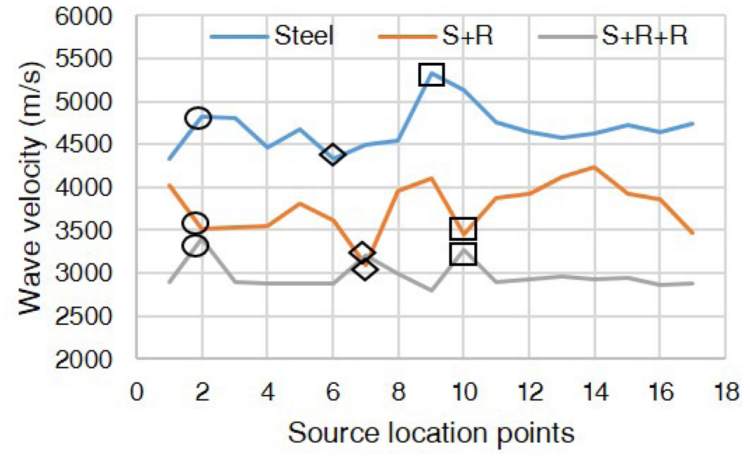

(a)

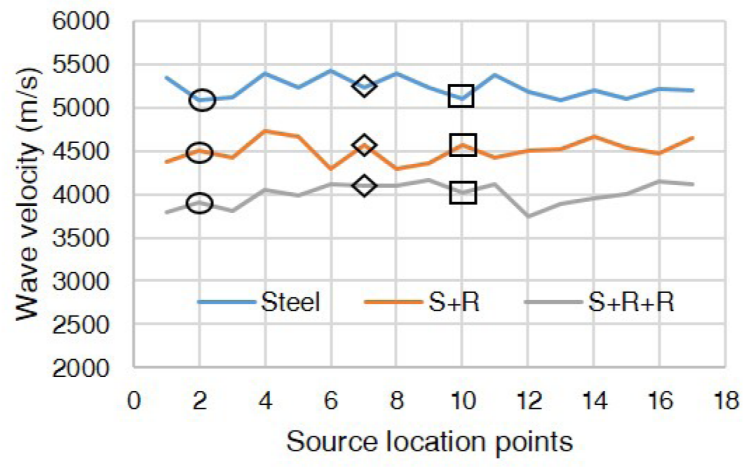

(b)

Figure 6. Velocity distribution at 17 source location points for the steel plate, steel plate covered with a single rubber layer $(\mathrm{S}+\mathrm{R})$ and the steel plate covered with a double rubber layer $(S+R+R)$ in case of (a) FTC method, (b) AIC method.

out of six contour lines, one line (black dashed line) did not intersect the point. However, an accurate source location was possible to identify by other intersecting contour lines.

\subsection{Comparison of Source Location Performance}

Two-dimensional source location measurements had been carried out at 17 different places (randomly selected) by both TOA and DOAT methods for all three cases of experiments, as mentioned earlier. Results were compared with the actual values of each PLB location and represented in Table 3, Table 4 and Fig. 9 respectively. Table 3 presents all DOAT results for all three cases by the number of the contour intersecting (Inter.) and the number of the contour non-intersecting (Non-inter.) point. It was already previously mentioned that the results of the DOAT method were calculated based on the six intersecting contour lines. Although theoretically, all six lines should intersect at the source location point; however, in the experimental results, all lines did not always coincide at one point. All of those observations are summarized in Table 3. However, despite these non-intersecting lines, the accuracy of source location at those points was not affected at all (Table 4), because accurate source locations were possible to identify by remaining intersecting contour lines. Table 4 represents the comparative results of source location among actual values, TOA in FTC method (TOA $\left.{ }_{\text {FTC }}\right)$, TOA in AIC method (TOA ${ }_{\text {AIC }}$ ) and DOAT methods in the case of the steel plate only. Similar results were also observed in the case of the steel plate covered 


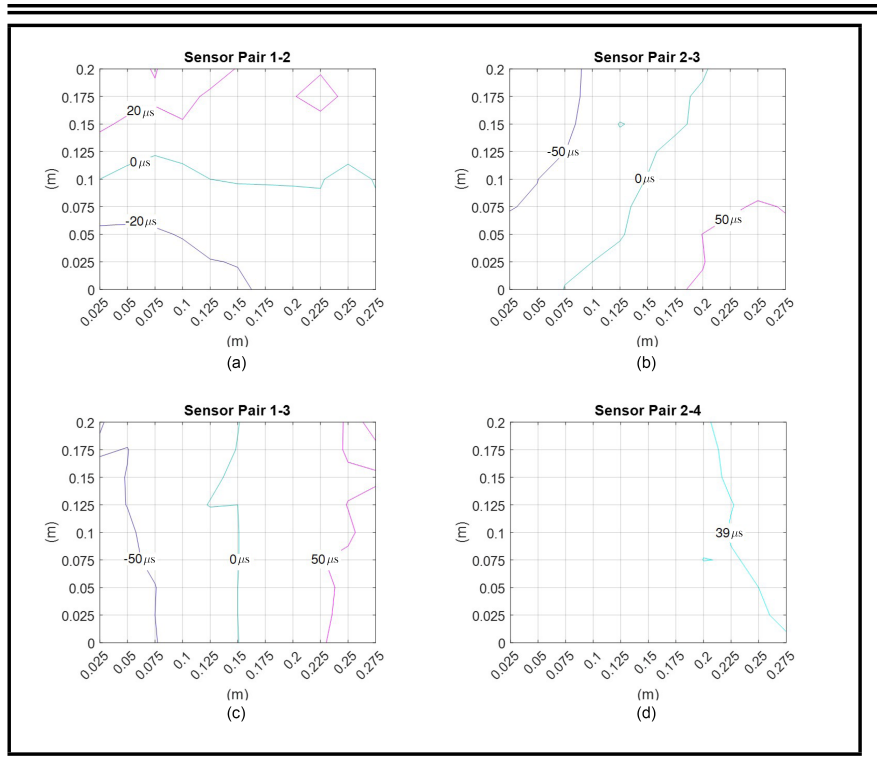

Figure 7. DOAT contour maps; (a) steel plate, (b) steel plate covered with a single rubber layer, (c) steel plate covered with a double rubber layer, and (d) DOAT $39 \mu$ s contour line for the sensor pair 2-4 of a specific AE event at node point 84 .

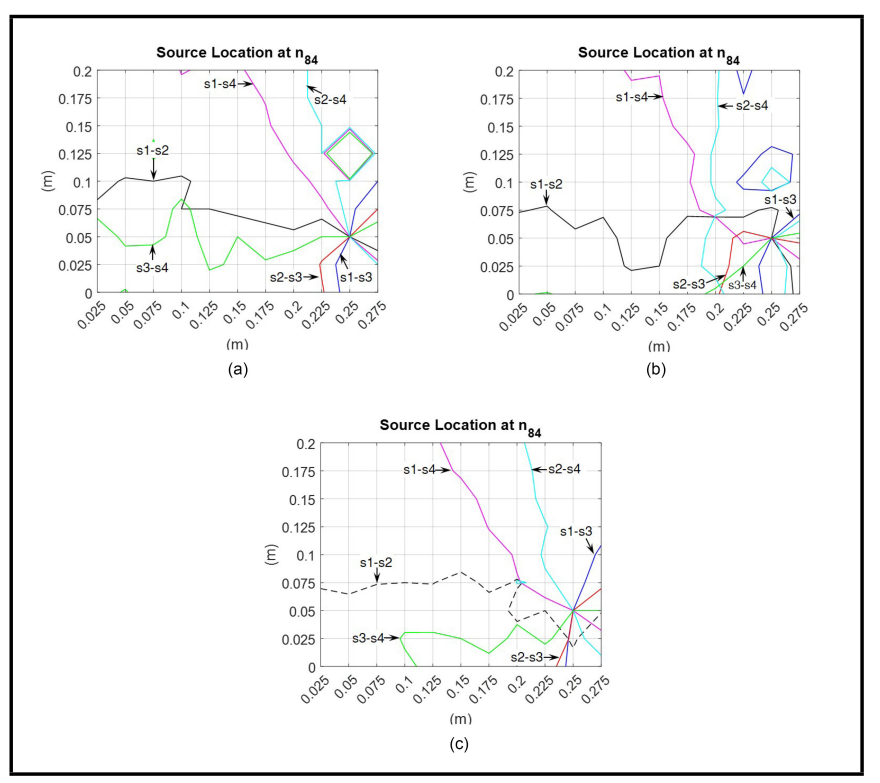

Figure 8. Source location using six DOAT contour maps at node point 84 in case of (a) steel plate, (b) steel plate covered with a single rubber layer, and (c) steel plate covered with a double rubber layer.

with a single rubber layer, and the steel plate covered with a double rubber layer and represented in Figs. 9(a)-(c). The localization points have been marked in these figures with the numerical values of 1 to 17 for a better understanding. Results show that errors were fluctuating from point to point for all cases in the TOA method. In those cases, location errors fluctuated from $0.17 \%$ to $44.76 \%$ in the case of steel, $0.39 \%$ to $52.72 \%$ in the case of steel covered with a single rubber layer and $1.98 \%$ to $50.84 \%$ in the case of steel covered with a

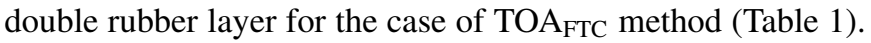
For $\mathrm{TOA}_{\mathrm{AIC}}$ method, location errors fluctuated from $0.19 \%$ to $37.66 \%$ in the case of steel, $0.41 \%$ to $34.38 \%$ in the case of steel covered with a single rubber layer and $1.06 \%$ to $35.78 \%$ in the case of steel covered with a double rubber layer (Table 2). It is already discussed that wave velocity is a crucial factor in the source location of the TOA technique. Therefore,

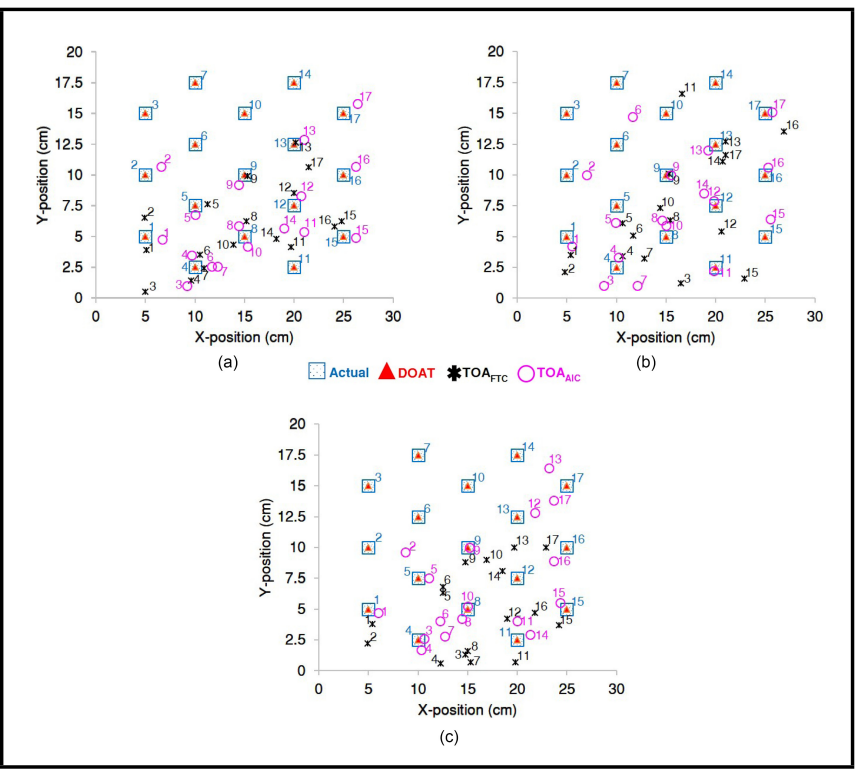

Figure 9. Comparison of source location among actual values, TOA ${ }_{F T C}$,

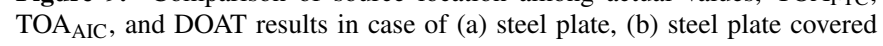
with a single rubber layer, and (c) steel plate covered with a double rubber layer.

due to structural inhomogeneity and complexity, wave velocity might be influenced, and hence, it was considered that the erroneous source locations are obtained in the TOA technique in the present research. However, DOAT is a velocity independent method, and thus, high accuracy in source location results were obtained even in the present complex structure.

Furthermore, the regression analysis had been carried out from the calculated 17 arbitrary points for getting the bestfit line, which is termed as the least-squares regression line $(L S R L)$, to predict the results throughout the test region of the present complex structure. Comparisons in $L S R L$ for $\mathrm{TOA}_{F T C}, \mathrm{TOA}_{\mathrm{AIC}}$ results and DOAT results along with actual values for all three cases of experiments are represented in Fig. 10. As it has been shown in Figs. 10(a)-(c) that the $L S R L$ for both TOA $F T C$, TOA AIC methods deviated from the actual line, whereas, $L S R L$ for DOAT method has not deviated. In both TOA $_{F T C}$, TOA AIC methods, the deviation occurred due to the errors in source locations although $L S R L$ for $\mathrm{TOA}_{\mathrm{AIC}}$ is less deviated (improved) than TOA $F T C$, whereas, no deviation was found in total DOAT source location calculations.

\section{CONCLUSIONS}

In this research, the performance of the AE source location has been analyzed in a complex multilayered structure by TOA and DOAT methods. The source location performance has been carried out at several places throughout the structure and compared with the actual values. Between the two methods, the DOAT method provides a better result in locating the source in a complex multilayered structure compared with the TOA method.

Time of arrival is a crucial factor for accuracy in the source location. Therefore, in the TOA method, the time of arrival has been verified based on FTC as well as AIC onset picking methods. It has been found that AIC onset picking improves the source location accuracy in TOA source location technique.

TOA method utilizes the velocity of the wave propagation directly. However, in a complex structure, the wave propaga- 
M. M. Hassan, et al.: PERFORMANCE INVESTIGATION OF TWO AE SOURCE LOCATION TECHNIQUES ON A PLANAR MULTILAYER...

Table 3. Summarized DOAT source location results for all three cases.

\begin{tabular}{|c|c|c|c|c|c|c|}
\hline \multirow[t]{2}{*}{ Location No. } & \multicolumn{2}{|c|}{ Steel } & \multicolumn{2}{|c|}{$S+R$} & \multicolumn{2}{|c|}{$\mathrm{S}+\mathrm{R}+\mathrm{R}$} \\
\hline & Contour Inter. & Contour Non-inter. & Contour Inter. & Contour Non-inter. & Contour Inter. & Contour Non-inter \\
\hline 1 & 6 & 0 & 5 & 1 & 6 & 0 \\
\hline 2 & 6 & 0 & 6 & 0 & 6 & 0 \\
\hline 3 & 4 & 2 & 6 & 0 & 6 & 0 \\
\hline 4 & 6 & 0 & 6 & 0 & 5 & 1 \\
\hline 5 & 5 & 1 & 6 & 0 & 6 & 0 \\
\hline 6 & 6 & 0 & 6 & 0 & 5 & 1 \\
\hline 7 & 6 & 0 & 6 & 0 & 6 & 0 \\
\hline 8 & 6 & 0 & 6 & 0 & 6 & 0 \\
\hline 9 & 6 & 0 & 6 & 0 & 6 & 0 \\
\hline 10 & 6 & 0 & 6 & 0 & 5 & 1 \\
\hline 11 & 6 & 0 & 3 & 3 & 6 & 0 \\
\hline 12 & 6 & 0 & 6 & 0 & 4 & 2 \\
\hline 13 & 6 & 0 & 6 & 0 & 4 & 2 \\
\hline 14 & 6 & 0 & 6 & 0 & 5 & 1 \\
\hline 15 & 6 & 0 & 6 & 0 & 5 & 1 \\
\hline 16 & 5 & 1 & 6 & 0 & 4 & 2 \\
\hline 17 & 4 & 2 & 5 & 1 & 5 & 1 \\
\hline
\end{tabular}

Table 4. Comparative results of source location among actual values, TOA $\mathrm{FTC}_{\text {, }}$ $\mathrm{TOA}_{\mathrm{AIC}}$ and DOAT methods in the case of the steel plate in coordinates $(\mathrm{X}, \mathrm{Y})$.

\begin{tabular}{|c|c|c|c|c|c|c|c|c||}
\hline $\begin{array}{c}\text { Location } \\
\text { No. }\end{array}$ & $\begin{array}{c}\text { Actual } \\
\text { Location } \\
{[\mathrm{cm}]}\end{array}$ & \multicolumn{2}{c|}{$\begin{array}{c}\text { TOAFTC } \\
\text { Location } \\
{[\mathrm{cm}]}\end{array}$} & \multicolumn{2}{c|}{$\begin{array}{c}\text { TOA } \\
\text { Location } \\
{[\mathrm{cm}]}\end{array}$} & \multicolumn{2}{c|}{$\begin{array}{c}\text { DOAT } \\
\text { Location } \\
{[\mathrm{cm}]}\end{array}$} \\
\hline & $\mathrm{X}$ & $\mathrm{Y}$ & $\mathrm{X}$ & $\mathrm{Y}$ & $\mathrm{X}$ & $\mathrm{Y}$ & $\mathrm{X}$ & $\mathrm{Y}$ \\
\hline 1 & 5 & 5 & 5.1 & 3.9 & 6.7 & 4.8 & 5 & 5 \\
\hline 2 & 5 & 10 & 4.9 & 6.5 & 6.6 & 10.7 & 5 & 10 \\
\hline 3 & 5 & 15 & 5 & 0.5 & 9.2 & 1 & 5 & 15 \\
\hline 4 & 10 & 2.5 & 9.6 & 1.4 & 9.7 & 3.5 & 10 & 2.5 \\
\hline 5 & 10 & 7.5 & 11.3 & 7.6 & 10 & 6.8 & 10 & 7.5 \\
\hline 6 & 10 & 12.5 & 10.5 & 3.5 & 11.7 & 2.6 & 10 & 12.5 \\
\hline 7 & 10 & 17.5 & 10.9 & 2.4 & 12.3 & 2.6 & 10 & 17.5 \\
\hline 8 & 15 & 5 & 15.2 & 6.2 & 14.4 & 5.9 & 15 & 5 \\
\hline 9 & 15 & 10 & 15.3 & 9.9 & 14.4 & 9.2 & 15 & 10 \\
\hline 10 & 15 & 15 & 13.9 & 4.3 & 15.3 & 4.2 & 15 & 15 \\
\hline 11 & 20 & 2.5 & 19.7 & 4.1 & 21 & 5.4 & 20 & 2.5 \\
\hline 12 & 20 & 7.5 & 20 & 8.5 & 20.7 & 8.3 & 20 & 7.5 \\
\hline 13 & 20 & 12.5 & 20.2 & 12.6 & 21 & 12.9 & 20 & 12.5 \\
\hline 14 & 20 & 17.5 & 18.2 & 4.8 & 19 & 5.7 & 20 & 17.5 \\
\hline 15 & 25 & 5 & 24.8 & 6.2 & 26.2 & 4.9 & 25 & 5 \\
\hline 16 & 25 & 10 & 24.1 & 5.8 & 26.2 & 10.7 & 25 & 10 \\
\hline 17 & 25 & 15 & 21.5 & 10.6 & 26.4 & 15.8 & 25 & 15 \\
\hline
\end{tabular}

tion path becomes complex due to the geometric complexity of the source location area, and thus, reduces source location performance.

DOAT method utilizes the arrival time differences only in source location calculation. Signal propagation velocity is not needed in this method, and thus, the DOAT method has become more accurate in all results.

In conclusion, according to the objectives of the present research, the DOAT source location technique has been identified as a relatively suitable technique for the application in source location to a complex multilayered structure.

\section{ACKNOWLEDGEMENT}

This work has been supported by JSPS KAKENHI Grant Number JP17K06266.

\section{REFERENCES}

1 Jihui, L. and Gang, Q. Improving source location accuracy of acoustic emission in complicated struc-

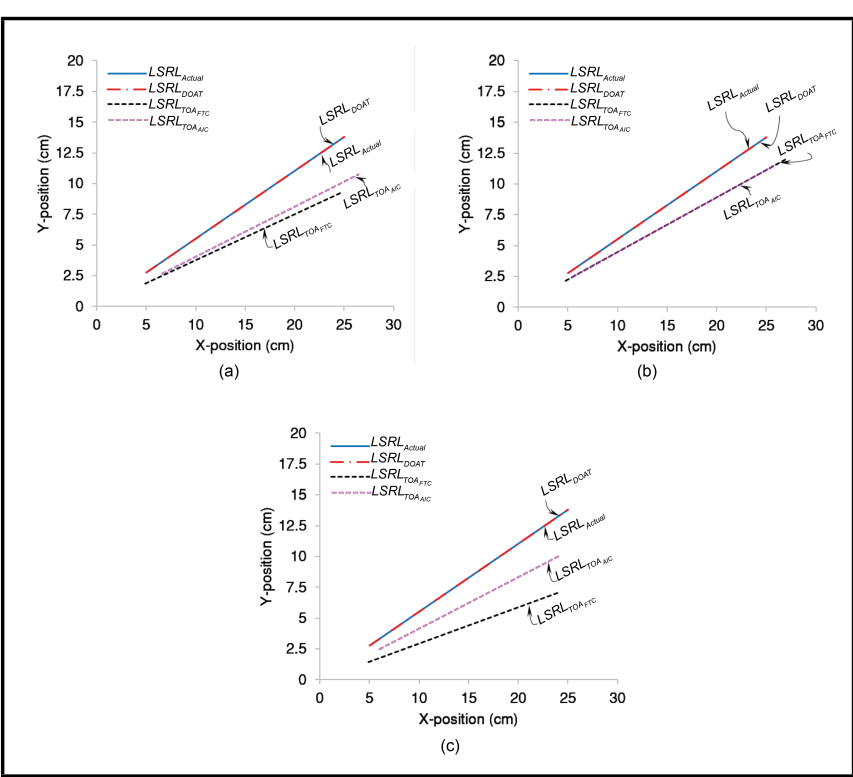

Figure 10. Comparative analysis of $L S R L$ among actual values, TOA ${ }_{F T C}$, $\mathrm{TOA}_{\mathrm{AIC}}$, and DOAT results in case of (a) steel plate, (b) steel plate covered with a single rubber layer, rubber and (c) steel plate covered with a double rubber layer.

tures, J. Nondestruct. Eval., 28(1), 1-8, (2009). https://dx.doi.org/10.1007/s10921-009-0042-z

2 Introduction to NDT, NDT Education Resource Center, Iowa State University, Retrieved from http://www.ndted.org (2001-2014).

3 Salinas, V., Vargas, Y., Ruzzante, J. and Gaete, L. Localization algorithm for acoustic emission, International Congress on Ultrasonics, Physics Procedia,3(1), 863-871, (2010). https://dx.doi.org/10.1016/j.phpro.2010.01.111

4 Ohtsu, M., Kaminaga, Y. and Munwam, M. C. Experimental and numerical crack analysis of mixed-mode failure in concrete by acoustic emission and boundary element method, Construction and Building Materials, 13(1-2), 57-64, (1999). https://dx.doi.org/10.1016/S09500618(99)00008-2

5 Dirk, A., Alex, C., Steve, W. and Karean, H. Acoustic emission source location in plate-like structures using a closely 
arranged triangular sensor array, J. Acoustic Emission, 28, 85-98, (2010).

6 Miller, K. R. and Hill, E. K. Nondestructive testing handbook, Acoustic emission testing, ASNT, USA (2005).

7 Khan, M.T.I., Sunichi, N. and Hasan, M. Structural damage localization by linear technique of acoustic emission, Open Journal of Fluid Dynamics, 4, 425-432, (2014). https://dx.doi.org/10.4236/ojfd.2014.45032

8 Tobias, A. Acoustic-emission source location in two dimensions by an array of three sensors, Non-Destructive Testing,9(1), 9-12, (1976).https://dx.doi.org/10.1016/00291021(76)90027-X

9 Schubert, F. Basic principles of acoustic emission tomography, 26th European Conference on Acoustic Emission Testing, (2004).

10 Baxter, M. G., Pullin, R., Holford, K. M. and Evans, S. L.: Delta T source location for acoustic emission, Mechanical Systems and Signal Processing, 21(3), 1512-1520, (2007). https://dx.doi.org/10.1016/j.ymssp.2006.05.003

11 Hsu, N. N. and Breckenridge, F. R. Characterization and calibration of acoustic emission sensors, Mater. Eval.,39(1), 60-68, (1981).

12 Markus, G. R. Investigation of pencil-lead breaks as acoustic emission sources, J. Acoustic Emission, 29, 184-196, (2011).

13 Dunegan, H.L. The DECI Report, An alternative to pencil lead breaks for simulation of acoustic emission signal sources, (2000).

14 Hamstad, M. A. Contrasts between the Acoustic Emission Signals Generated by Monopole Versus Dipole Sources, Advanced Materials Research, 13-14, 61-68, (2006). https://dx.doi.org/10.4028/www.scientific.net/AMR.1314.61

15 Sedlak, P., Hirose, Y., Enoki, M. and Sikula, J. Arrival time detection in thin multilayer plates on the basis of akaike information criterion, J. Acoustic Emission, 26, 182-188, (2008).

16 Kitagawa, G., Akaike, H. A procedure for the modeling of non-stationary time series, Ann. Inst. Statist. Math., 21, 407-419, (1978).

17 Sleeman, R. and van Eck, T. Robust automatic Pphase picking: an on-line implementation in the analysis of broadband seismogram recordings, Phys. Earth Planet Inter., 113(1-4), 265-275, (1999). https://dx.doi.org/10.1016/S0031-9201(99)00007-2

18 Nivesrangsan, P., Steel, J. A. and Reuben, R. L. Source location of acoustic emission in diesel engines, Mechanical Systems and Signal Processing, 21(2), 1103-1114, (2007). https://dx.doi.org/10.1016/j.ymssp.2005.12.010

19 Gang, Y. and Jianfei, T. A Bayesian approach for localization of acoustic emission source in plate-like structures, Mathematical Problems in Engineering, 2015, 1-14, (2014). https://dx.doi.org/10.1155/2015/247839
20 Dong, L., Sun, D., Li, X. and Du, A. K. Theoretical and experimental studies of localization methodology for $\mathrm{AE}$ and microseismic sources without pre-measured wave velocity in mines, IEEE Access, 5, 16818-16828, (2017). https://dx.doi.org/10.1109/ACCESS.2017.2743115

21 Dong, L., Zou, W., Li, X., Shu, W. and Wang, Z. Collaborative localization method using analytical and iterative solutions for microseismic/ acoustic emission sources in the rockmass structure for underground mining, Engineering Fracture Mechanics, 210, 95-112, (2019). https://dx.doi.org/10.1016/j.engfracmech.2018.01.032

22 Rijn, N.L. Investigating the behaviour of acoustic emission waves near cracks: using the finite element method, Delft University of Technology, (2017).

23 Sedlak, P., Hirose, Y. and Enoki, M. Acoustic emission localization in thin multi-layer plates using first-arrival determination, Mechanical Systems and Signal Processing, 36, 636-649, (2013). https://dx.doi.org/10.1016/j.ymssp.2012.11.008

24 Akaike, H. Information theory and an extension of the maximum likelihood principle, 2nd International Symposium of Information Theory, B. N. Petrov and F. Csaki, eds., Akademiai Kiado, Budapest, 267-281, (1973).

25 Maeda, N. A method for reading and checking phase times in autoprocessing system of seismic wave data, Zisin, 38, 365-379, (1985). https://dx.doi.org/10.4294/zisin1948.38.3_365

26 Khan, T. I., Hassan, M. and Takata, R. Effect of wave velocity in two-dimensional AE damage location on a steel plate, International Journal of COMADEM, 20(2), (2017).

27 Noorsuhada, M. N., Norazura, M. B., Azmi, I., Hamida, M. S., Shahidan, S. and Saliah, S. N. An investigation of an acoustic wave velocity in a reinforced concrete beam from out of plane and in plane sources, INTECH (2013). https://dx.doi.org/10.5772/54448

28 Norazura, M. B., Pullin, R., Holford, K. M. and Lark, R. J. A practical investigation into acoustic wave propagation in concrete structures, $A d$ vanced Materials Research, 13-14, 205-212, (2006). https://dx.doi.org/10.4028/www.scientific.net/AMR.1314.205

29 Li, Q., Dong, L., Li, X., Yin, Z. and Liu, X. Effects of sonic speed on location accuracy of acoustic emission sources in rocks, Trans. Nonferrous Met. Soc. China, 21, 2719-2726, (2011). https://dx.doi.org/10.1016/S10036326(11)61115-1

30 Nondestructive Inspection Series, Acoustic Emission Testing 1, Japan Society for Nondestructive Inspection, (2006).

31 Steven, J. The two-dimensional source location problem for time differences of arrival at minimal element monitoring arrays, J. Acoust. Soci. Am., 121(6), 3579-3594, (2007). https://dx.doi.org/10.1121/1.2734404 